\title{
Experimental measurement of time-dependant photon scatter for diffuse optical tomography
}

\author{
Niksa Valim \\ James Brock \\ Mark Niedre \\ Northeastern University \\ Department of Electrical and Computer Engineering \\ Dana Research Center, Boston, Massachusetts 02115
}

\begin{abstract}
Time-resolved measurement of early arriving photons through diffusive media has been shown to effectively reduce the high degree of light scatter in biological tissue. However, the experimentally achievable reduction in photon scatter and the impact of time-gated detection on instrument noise performance is not well understood. We measure time-dependent photon density sensitivity functions (PDSFs) between a pulsed laser source and a photomultiplier tube operating in timecorrelated single-photon-counting mode. Our data show that with our system, measurement of early arriving photons reduces the full width half maximum of PDSFs on average by about 40 to $60 \%$ versus quasicontinuous wave photons over a range of experimental conditions similar to those encountered in small animal tomography, corresponding to a 64 to $84 \%$ reduction in PDSF volume. Factoring in noise considerations, the optimal operating point of our instrument is determined to be about the $10 \%$ point on the rising edge of the transmitted intensity curve. Time-dependant Monte Carlo simulations and the time-resolved diffusion approximation are used to model photon propagation and are evaluated for agreement with experimental data. ( 2010 Society of PhotoOptical Instrumentation Engineers. [DOI: 10.1117/1.3523371]
\end{abstract}

Keywords: diffuse optical tomography; time-resolved; early photons; Monte Carlo.

Paper 10469R received Aug. 23, 2010; revised manuscript received Oct. 8, 2010; accepted for publication Oct. 18, 2010; published online Nov. 30, 2010.

\section{Introduction}

Diffuse optical tomography (DOT) and fluorescence-mediated tomography (FMT) are emerging as valuable tools for noninvasive visualization of native tissue chromophores and fluorescent markers in humans and in laboratory animals. ${ }^{1-3}$ These have been used in a wide range of biomedical applications from clinical-scale breast, brain, and joint imaging ${ }^{4-6}$ to preclinical small animal molecular imaging with targeted fluorescent probes and fluorescent proteins. ${ }^{7-12}$ Briefly, both DOT and FMT involve (1) illumination of the target tissue volume with a light source, (2) measurement of the fluorescent and excitation light transmitted through the tissue, (3) modeling of light propagation through diffusive tissue, and (4) solving the resulting inverse problem to obtain the tomographic image. ${ }^{13}$

Among the important technical challenges in DOT and FMT is the relatively poor resolution that results from the high degree of light scatter in biological tissue. Time-resolved measurement of early arriving photons has been demonstrated by a number of groups to effectively reduce photon scatter. ${ }^{14-24}$ Specifically, early photons take more direct paths between a given source and detector pair than un-gated, continuous wave $(\mathrm{CW})$ photons. The extent of photon scatter and hence the volume of the instrument photon density sensitivity function (PDSF) is therefore reduced, improving the condition number of the inverse problem. ${ }^{15}$

Address all correspondence to: Mark Niedre, Northeastern University, Department of Electrical and Computer Engineering, Dana Research Center, Boston, Massachusetts 02115. Tel: 617-373-5410; Fax: 617-373-8970. E-mail: mniedre@ece.neu.edu.
Although this concept has been demonstrated previously, significant details with respect to measurement of early photons in tomographic imaging remain poorly understood, for example, (1) what quantitative reduction in the instrument PDSF can be obtained by measuring early arriving photons under typical experimental conditions? (2) how sensitive is the improvement to the specific detection time gate used? (3) how sensitive is the improvement to the sample optical properties and optical path length? and (4) what is the trade-off between early time-gated imaging and instrument noise performance?

In this work, we measured time-dependant instrument PDSFs through liquid tissue-simulating optical phantoms using a picosecond-pulsed laser source and a multianode photomultiplier tube (PMT) module operating in time-correlated singlephoton-counting (TCSPC) mode. We demonstrated that the full width half maximum (FWHM) of the instrument PDSF could be reduced on average by a factor of 40 to $60 \%$ by measuring photons that arrived during the 5 to $10 \%$ point on the rising edge of the transmitted intensity curve. This was demonstrated over a range of optical properties and path lengths relevant for red and near-infrared (NIR) small animal imaging. We also show that these data agree well with the results of time-dependant Monte Carlo simulations except at very early time points where simulations predict that a larger improvement could potentially be obtained with a modified instrument. In contrast, results from the time-resolved diffusion approximation to the Boltzmann transport equation (BTE) showed good experimental agreement for quasi-CW measurements but significant disagreement at early

$1083-3668 / 2010 / 15(6) / 065006 / 8 / \$ 25.00$ (C) 2010 SPIE 

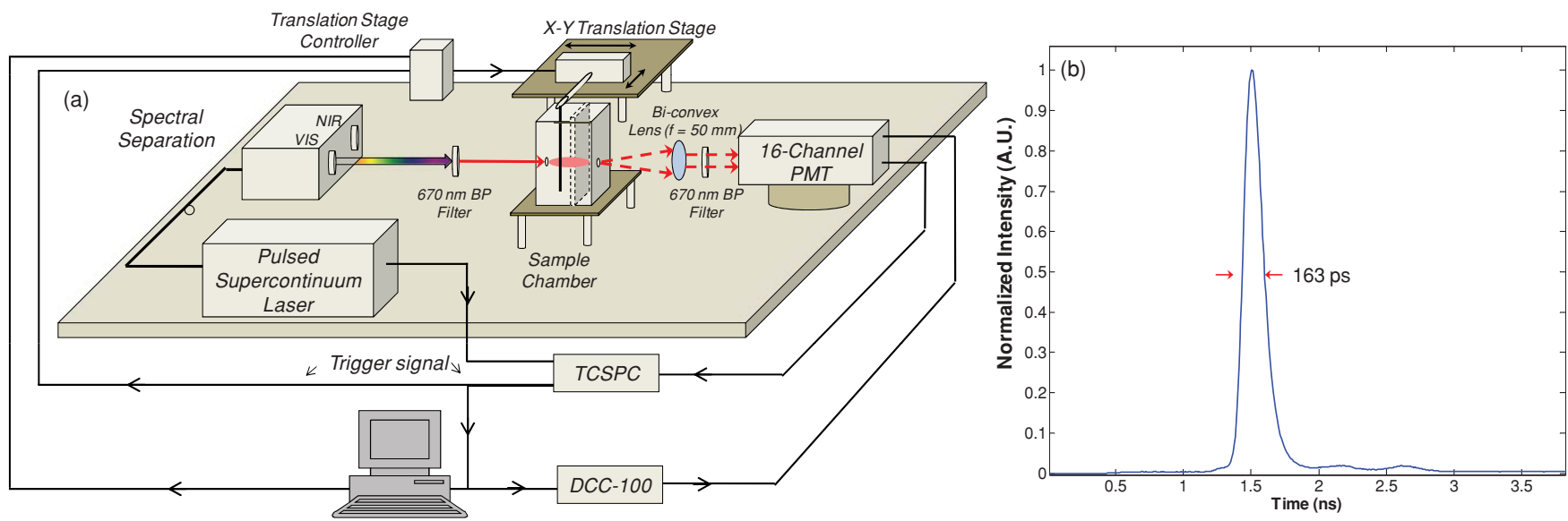

Fig. 1 (a) Schematic of the system used to measure time-dependant PDSFs and (b) the FWHM of the system temporal impulse response function was 163 ps.

time gates. These results are important for time-dependent FMT and DOT since they demonstrate that a 64 to $84 \%$ reduction in instrument PDSF volume can be practically obtained with a corresponding improvement in imaging resolution. To our knowledge, this has never been demonstrated quantitatively previously with a systematic experimental approach.

\section{Methods and Materials}

\subsection{Instrumentation}

The system used for time-resolved measurement of instrument PDSFs is shown in Fig. 1(a). A customized pulsed supercontinuum fiber laser with an $80-\mathrm{MHz}$ repetition rate was used as the light source (Koheras SuperK Power, NKT Photonics, Birkerod, Denmark). The laser was modified by the manufacturer so that it had a shortened fiber compared to standard models, which reduced the pulse width to approximately $300 \mathrm{ps}$. The output of the broadband laser was passed through a visible-NIR splitter device (SpectraK Split; NKT Photonics) to isolate the 550- to $850-\mathrm{nm}$ section of the pulse and then was further filtered with a 670-nm bandpass (BP) interference filter (30-nm FWHM; Chroma Technology, Bellows Falls, Vermont). It is a property of supercontinuum pulse generation that different temporal positions in the broadband output pulse correspond to different emission wavelengths; therefore, wavelength selection with the interference filter reduced the pulse width to about 30 ps (i.e., reduced by a factor of the ratio of the filter BP divided by the full pulse spectral width).

The $670-\mathrm{nm}$ light was focused to a $1-\mathrm{mm}$ spot size at the surface of the sample chamber with a 300-mm-focal-length achromatic planoconvex lens (Edmund Optics, Barrington, New Jersey). The width of the custom-made chamber could be adjusted between 1.5 and $6 \mathrm{~cm}$ and the chamber was filled with liquid media approximating the optical properties of biological tissue at red and NIR wavelengths (see Sec. 2.2). A 1-mm pinhole was placed at the exit plane of the chamber, coaxial with the input position of the laser. Light from the chamber was collimated with a planoconvex lens ( $f=50 \mathrm{~mm}$; Edmund) and passed through a second $670-\mathrm{nm}$ interference filter.

Transmitted light was measured with a 16-channel photomultiplier tube assembly with a large $16-\times 16-\mathrm{mm}$ active cath- ode area (PML-16-C, Becker and Hickl, Berlin, Germany) connected to a TCSPC card (SPC-130, Becker and Hickl). The temporal resolution (i.e., time bin width) of the TCSPC card was set to $7 \mathrm{ps}$ in all cases. The PMT assembly was preselected by the manufacturer to have an impulse response time of about $150 \mathrm{ps,}$ suitable for photon counting. For the current experiments, the signals from each channel were summed after correction for the minor time skew between channels (about \pm 75 ps per channel) to yield a single measurement. In future experiments, we intend to treat the 16 channels independently, for example, by adding spectroscopic capabilities to our system. The measured instrument temporal impulse response function (TIRF) is shown in Figure 1(b). The FWHM of the TIRF was 163 ps, which agrees well the performance specifications of our laser source $(\sim 30 \mathrm{ps})$ and multichannel PMT $(\sim 150 \mathrm{ps})$.

\subsection{Optical Phantoms and Data Collection}

Homogeneous liquid optical phantoms were prepared from a mixture of $20 \%$ Intralipid stock solution (Baxter Healthcare Corporation, Deerfield, Illinois) and ink (Higgins Ink, Sanford Corporation, Bellwood, Illinois), which modified the scattering and absorption coefficients of the solution, respectively. Optical properties were varied over a range that is relevant for red and NIR imaging in mice; ${ }^{25,26}$ specifically the final intralipid concentration was varied from 1 to $5 \%$ (corresponding to approximately $\mu_{s}^{\prime}=17$ to $85 \mathrm{~cm}^{-1}$ at $670 \mathrm{~nm}$ ) and the final ink concentration was varied ${ }^{25}$ from 50 to $150 \mathrm{ppm}$ (corresponding to approximately $\mu_{a}=0.15$ to $0.45 \mathrm{~cm}^{-1}$ at $670 \mathrm{~nm}$ ). Measurements were repeated at least four times for each condition.

A 1-mm-diam absorbing rod was translated through the medium $(x$ stepsize $=0.25 \mathrm{~mm}, z$ stepsize $=0.5 \mathrm{~mm})$ using computer-controlled stepper motor stages (XSlide, Velmex Inc., Bloomfield, New York) and the transmitted full-time curve was recorded at each point. This enabled us to measure the relative effect of the absorber at each position for each time gate. Acquisition times were $2 \mathrm{~s}$ per position and the laser power was adjusted between 0.5 and $10 \mathrm{~mW}$ at the sample to give comparable photon count rates for each condition.

To measure the fluorescence PDSF, the absorbing rod was replaced with a 2-mm-diam $\times 1$-cm-long tube filled with Intralipid (1\% Intralipid with $50 \mathrm{ppm}$ ink) and $10-\mu \mathrm{M}$ Alexafluor-680 dye 
(Invitrogen Corporation, Carlsbad, California) attached to the end of a fine rod. The fluorescent tube was positioned so that the middle was at the same vertical position as the laser. In this case, the filter in front of the PMT was replaced with a 730-nm filter (30-nm BP; Chroma) to detect the fluorescent light.

\subsection{Data Analysis}

Full time curves were first acquired for each experimental condition without the absorbing rod in place. In each case, curves were analyzed to find time points corresponding to $0.5,1,5,10$, and $50 \%$, on the rising and falling edges of the measured timeresolved curves as well as the peak and quasi-CW (integrated) intensities. The rationale here was that this enabled comparison of data for different optical properties and chamber path lengths (as opposed to absolute time gates), since the length of the full time curves vary significantly between conditions. Signals for each rod position and each time point within a narrow (7 ps) time window were normalized to those from the control signals (i.e., without an absorbing rod in place) and then subtracted from unity. These data were then plotted as a function of rod position to yield the experimental PDSF. Data were then analyzed to determine the FWHM in the middle plane of the PDSF for each condition and time gate.

\subsection{Analytical Calculation of PDSFS}

Analytical calculation of time-dependent PDSFs has been comprehensively described previously in the literature. The photon field at a time $t$ between a source and detector pair following a laser pulse can be written as the weight function: ${ }^{18}$

$$
W\left(r_{s}, r_{d}, t\right)=\iint_{0}^{t} U_{o}\left(r, r_{s}, \tau\right) G\left(r_{d}, r, t-\tau\right) \mathrm{d} \tau \mathrm{d}^{3} r,
$$

where $\tau$ is an integrating factor for time; $r$ is the position inside the diffuse media; and $r_{s}$ and $r_{d}$ are the source and detector positions of each source-detector pair, respectively. The Green's function $G$ and $U_{o}$ are normally calculated using an analytical approximation to the BTE. In this work, we used the diffusion approximation to the BTE, since it is commonly used in modeling light propagation in diffusive media, for example, in small animal tomographic imaging. Importantly, diffusion theory uses the P-1 approximation to the spherical harmonic expansion of the photon radiance and also assumes that all sources emit isotropically. ${ }^{27}$ Using the diffusion approximation, it can be shown that the time-resolved photon fluence rate following an infinitely short laser pulse in an infinite medium is ${ }^{28}$

$$
N(r, t)=\frac{1}{(4 \pi D c t)^{3 / 2}} \exp \left(\frac{-r^{2}}{4 D c t}\right) \exp \left(-\mu_{a} t\right),
$$

where $t$ is the time following the pulse; $r$ is the position in the media; $D$ is the diffusion coefficient, where $D=\left[3\left(\mu_{s}^{\prime}+\mu_{a}\right)\right]^{-1}$; $\mu_{s}^{\prime}$ is the reduced scattering coefficient; $\mu_{a}$ is the absorption coefficient; and $c$ is the speed of light in the media.

\subsection{Monte Carlo Simulations}

We developed a set of C-code to calculate time-resolved PDSFs based on the Monte Carlo code publically available on the Oregon Medical Laser Center website. ${ }^{29}$ Semiinfinite slab geome- tries of varying thickness $(l)$, scatter $\left(\mu_{s}\right)$, and absorption $\left(\mu_{a}\right)$ were used to model our experimental conditions. In all cases, the anisotropy factor $g$ for Intralipid was taken to be 0.85 . Photons were launched into the media from a pencil beam source, and the scattered photon path and exit time was logged for all photons that exited the media at a 1- $\times 1-\mathrm{mm}$ "detector" position coaxial from the entry point. In each case, 1 billion photons were tracked, which required about $72 \mathrm{~h}$ of computation on a $3.2-\mathrm{GHz}$ Dual-Core PC running Linux. The paths of photon propagation for the rising edge of the transmitted intensity curve were then analyzed to yield the PDSFs.

\section{Results}

\subsection{Analysis of PDSFs at Different Time Gates}

The basic analysis performed in these experiments is shown in Fig. 2. As already described above, full-time transmitted intensity curves were analyzed to yield time points corresponding to $0.5,1,5,10$, and $50 \%$ of the peak intensity on the rising and falling edges of the curve. A typical transmitted full time curve for a $2-\mathrm{cm}$ chamber path length with $1 \%$ Intralipid and $50 \mathrm{ppm}$ ink (corresponding to $\mu_{s}^{\prime}=17 \mathrm{~cm}^{-1}$ and $\mu_{a}=0.15 \mathrm{~cm}^{-1}$ ) is shown in Fig. 2(a). These data were analyzed, as already described, to yield the PDSF at each time point. Example measured PDSFs at the $50 \%$ rise, peak, and $50 \%$ decay points along the curve are shown in Figs. 2(b) to 2(d), respectively. As expected, the plots became broader with time due to the photon scatter following the laser pulse.

As a metric of the extent of this photon scatter, the FWHM of the PDSF at the middle plane (i.e., where photon scatter was maximal) of the sample chamber was measured. The FWHM of the PDSF as a function of time gate is shown in Fig. 2(e). In this case, the FWHM of PDSFs for time gates earlier than the $10 \%$ rise point of the TPSF was $48 \pm 8 \%$ of the quasi-CW width. Since this represents the reduction in the PDSF radius, the corresponding reduction in the 3-D PDSF volume was therefore approximately $77 \%$. The quasi-CW FHWM measurement here corresponds to the first moment of the time-resolved curve in Fig. 1(a) (as opposed to the peak), so that the peak of the curve is actually slightly "early" compared to the quasi-CW measurement. Photons arriving later than the peak of the TPSF had a larger PDSF FWHM compared to quasi-CW photons. The absolute photon arrival times were difficult to measure but the $0.5 \%$ rise point was approximately $180 \mathrm{ps}$ after the laser pulse entered the front surface sample chamber.

\subsection{Effect of Chamber Path Length}

To test the effect of the path length on the width of the PDSF, the width of the sample chamber was varied from 1.5 to $6 \mathrm{~cm}$. As in the preceding, the chamber was filled with liquid solution containing $1 \%$ Intralipid and $50 \mathrm{ppm}$ ink (corresponding to $\mu_{s}^{\prime}=17 \mathrm{~cm}^{-1}$ and $\mu_{a}=0.15 \mathrm{~cm}^{-1}$ ). The absolute FWHM of the PDSF in millimeters is shown in Fig. 3(a), along with the relative PDSF FWHM [Fig. 3(b)] for the 5 and $50 \%$ rise points of the transmitted curve. As expected, increasing the chamber path length in both cases resulted in a wider PDSF since more scatter occurs through the longer path length. However, the relative width of the PDSF did not vary significantly, particularly 

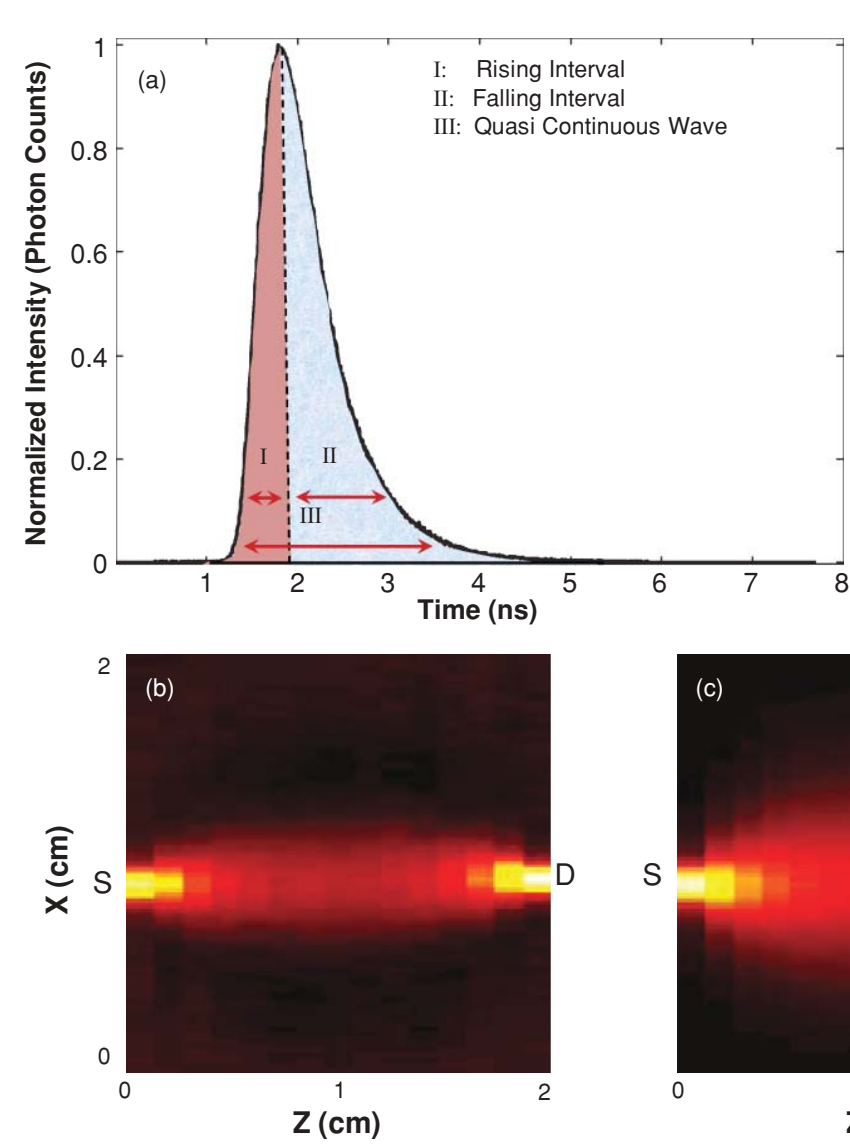

Percent of Peak Transmitted Intensity

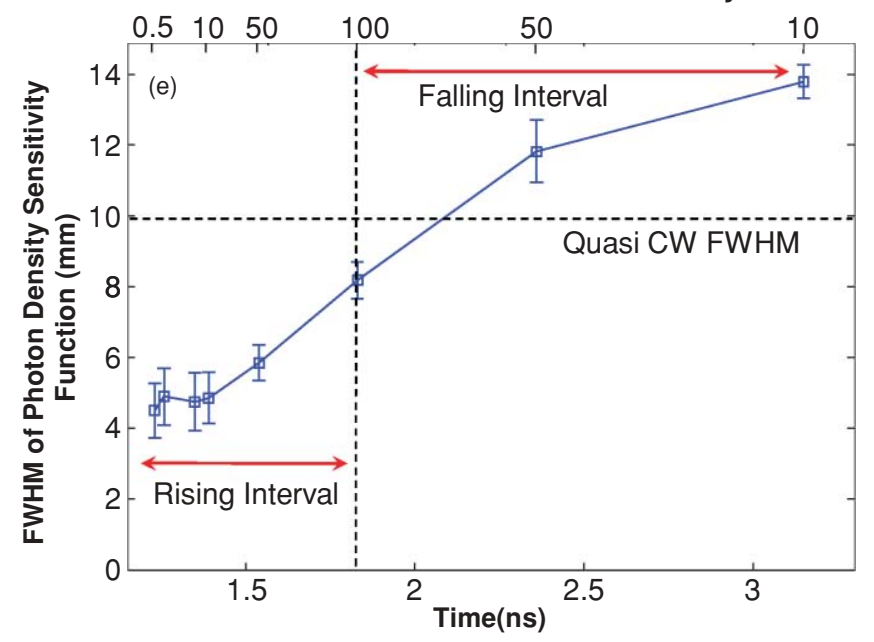

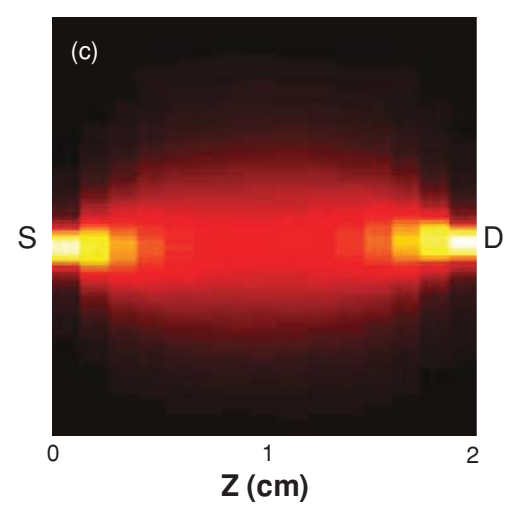

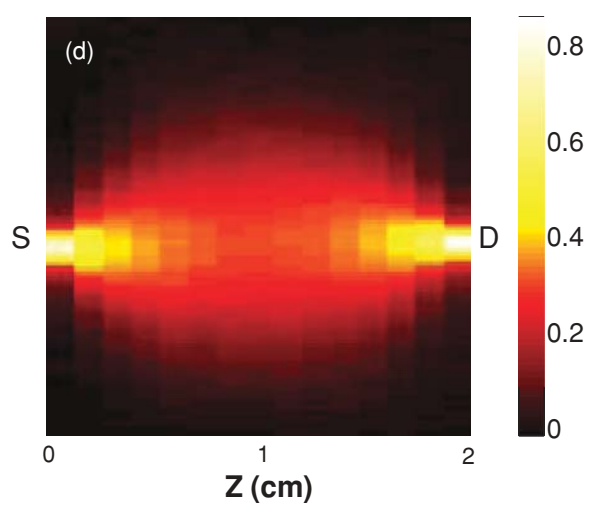

Fig. 2 (a) Measured time-resolved intensity for a pulse transmitted through a 2-cm-wide chamber filled with a homogeneous solution of $1 \%$ Intralipid and $50 \mathrm{ppm}$ ink. The curve was analyzed at each rod position to give the instrument PDSF at different time points (see text for details). Example PDSFs at (b) the $50 \%$ point on the rising edge of the transmitted time-resolved curve, (c) the peak of the curve, (d) and the $50 \%$ point on the falling edge of the time-resolved curve. (e) The FWHM of the PDSF as a function of measurement time. Reduction of PDSF FWHM of more than 50\% was observed for early photons versus quasi-CW photons.
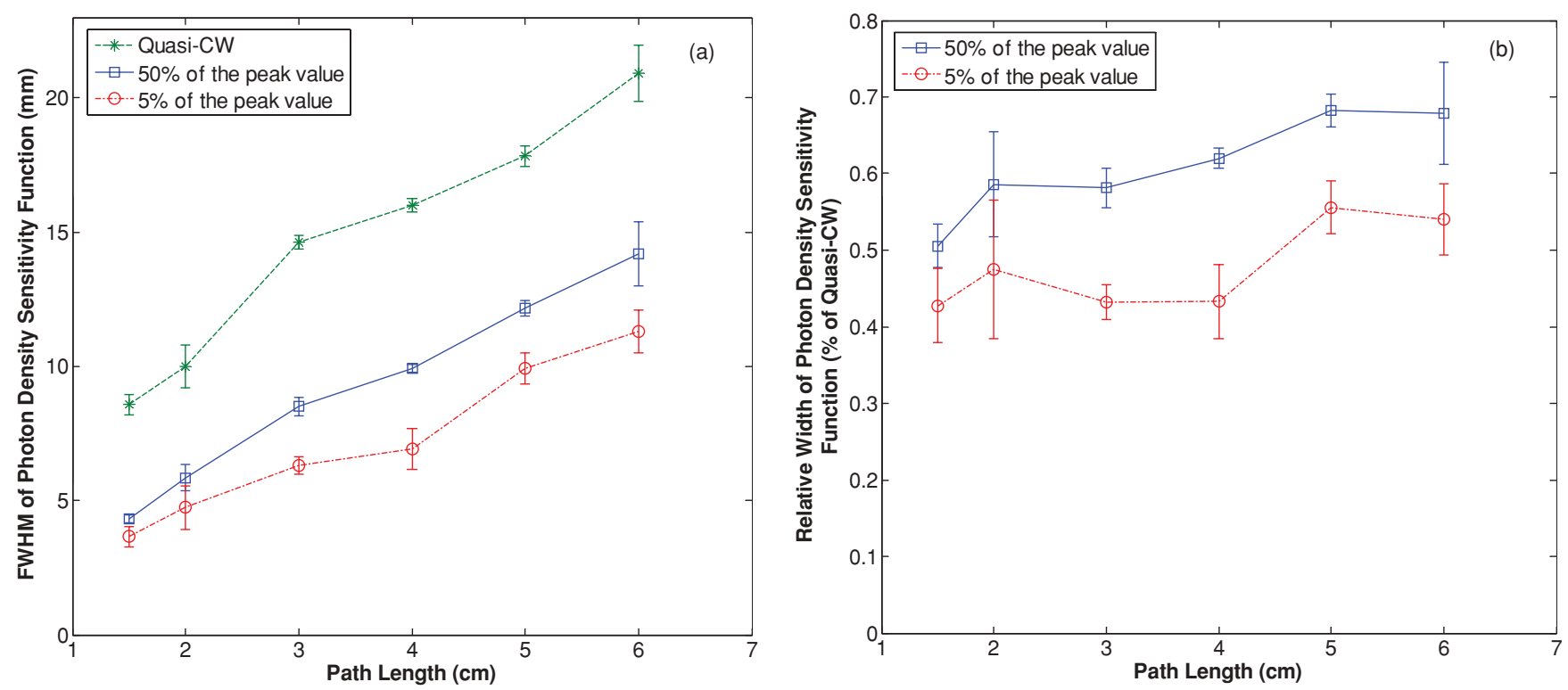

Fig. 3 Effect of chamber path length on (a) the absolute PDSF FWHM at different time gates and (b) the relative FWHM of the PDSF time gate at 5 and $50 \%$ points on the rising edge of the full-time curve compared to the quasi-CW PDSF FWHM. In all cases $1 \%$ Intralipid and 50 ppm ink were used. 

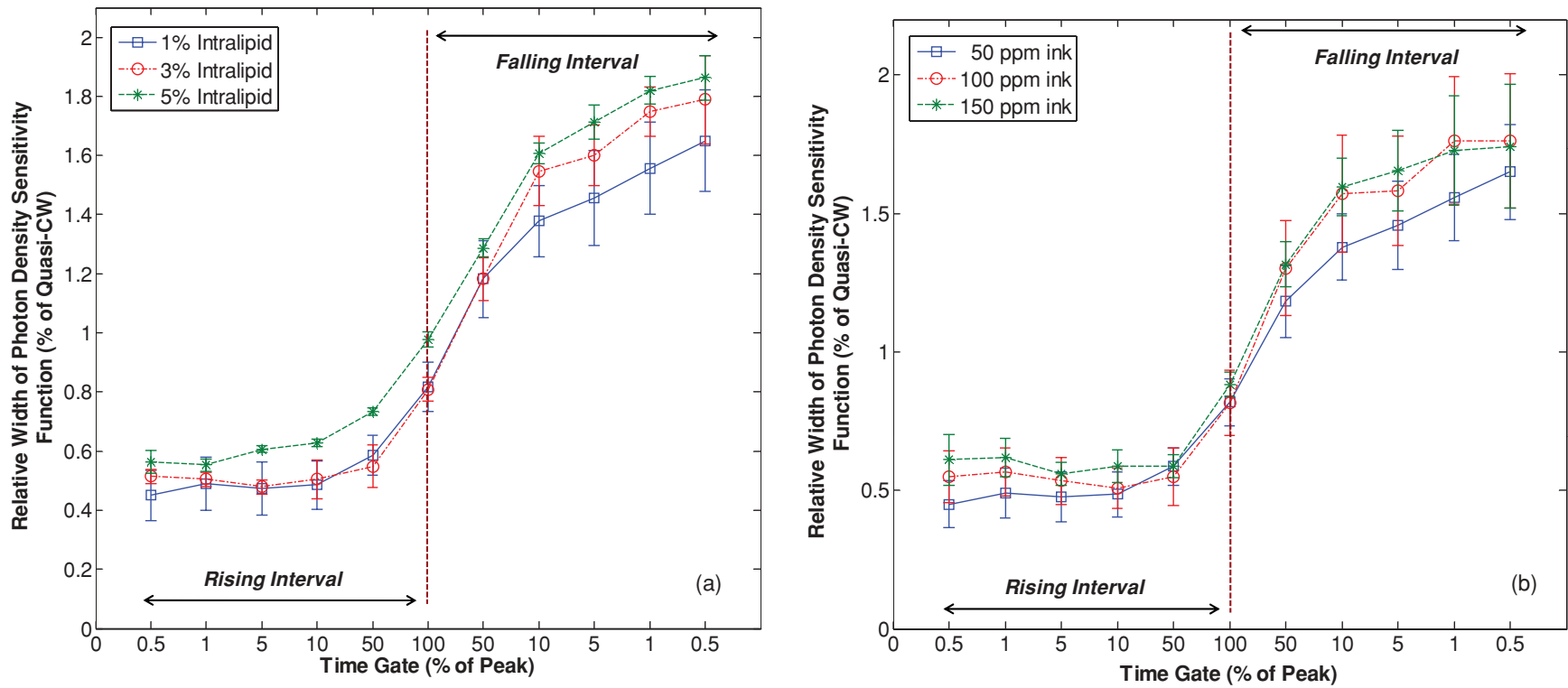

Fig. 4 Effect of (a) changing the Intralipid concentration (ink concentration was 50 ppm in all cases) and (b) changing the ink concentration (Intralipid concentration was $1 \%$ in all cases) on the relative PDSF FWHM at different time points.

for chamber path lengths between 1.5 and $4 \mathrm{~cm}$. Specifically, the PDSF FWHM was 42 to $48 \%$ and 50 to $58 \%$ of the quasi-CW PDSF for the 5 and $50 \%$ points on the rise portion of the curve, respectively, for 1.5 - to $4-\mathrm{cm}$ chamber widths. Chamber path lengths longer than $4 \mathrm{~cm}$ resulted in slightly broader relative PDSF FHWMs at early time gates.

\subsection{Effect of Media Optical Properties}

To understand the influence of phantom optical properties on the FWHM of the measured PDSF, we varied $\mu_{s}^{\prime}$ and $\mu_{a}$ over a range meant to mimic optical properties found in biological tissues at red and NIR wavelengths. Figure 4(a) shows the effect on the relative FWHM of the measured PDSFs for 1, 3, and $5 \%$ Intralipid $\left(\mu_{s}^{\prime}=17,34\right.$, and $81 \mathrm{~cm}^{-1}$, respectively) with 50 ppm ink added $\left(\mu_{a}=0.15 \mathrm{~cm}^{-1}\right)$ in call cases. When scattering was increased, the absolute width of the FWHM decreased, but the relative width of the FWHM for early arriving photons compared to quasi-CW photons increased slightly from $48 \pm 8$ to $60 \pm 2 \%$. The decrease in the absolute PDSF FWHM with increasing scatter results from a corresponding reduction in the diffusion coefficient $D$ and a more-rapid radial fall-off of the fluence rate from the source, as in Eq. (2). Similarly, Fig. 4(b) shows the absolute width of the FWHM decreased when the absorption coefficient was increased, whereas the relative width increased from about $48 \pm 8$ to $56 \pm 4 \%$ of the quasi-CW PDSF. Therefore, larger optical properties resulted in slightly less reduction in photon scatter when measuring early photons.

\subsection{Fluorescence PDSF}

The absorbing rod was then replaced with a 2-mm-diam tube filled with $10 \mu \mathrm{M}$ Alexafluor-680 dye in Intralipid. For these experiments, the chamber was filled with a solution of $1 \%$ Intralipid with $50 \mathrm{ppm}$ ink $\left(\mu_{s}^{\prime}=17 \mathrm{~cm}^{-1}\right.$ and $\left.\mu_{a}=0.15 \mathrm{~cm}^{-1}\right)$. The measured FWHM of the PDSF as a function of the quasiCW fluorescent signal is shown in Fig. 5. Although not indicated here, the length of the measured fluorescence curve was significantly longer than the excitation curve due to convolution with the 1.2-ns fluorescence lifetime. In this case, the PDSF FWHM at early gates was $52 \pm 6 \%$ of that for quasi-CW photons. The reduction was therefore slightly less than was observed in the case of the absorbing rod. It is known that the scattering coefficient for Intralipid (as well as biological tissue) decreases with increasing wavelength so that the contribution of the scatter at the emitted wavelength $(730 \mathrm{~nm})$ may have slightly increased the width of the PDSF versus the excitation light $(670 \mathrm{~nm})$. Nevertheless, the reduction in the measured fluorescent PDSF at early gates was observed to be similar to that for the absorption PDSF.

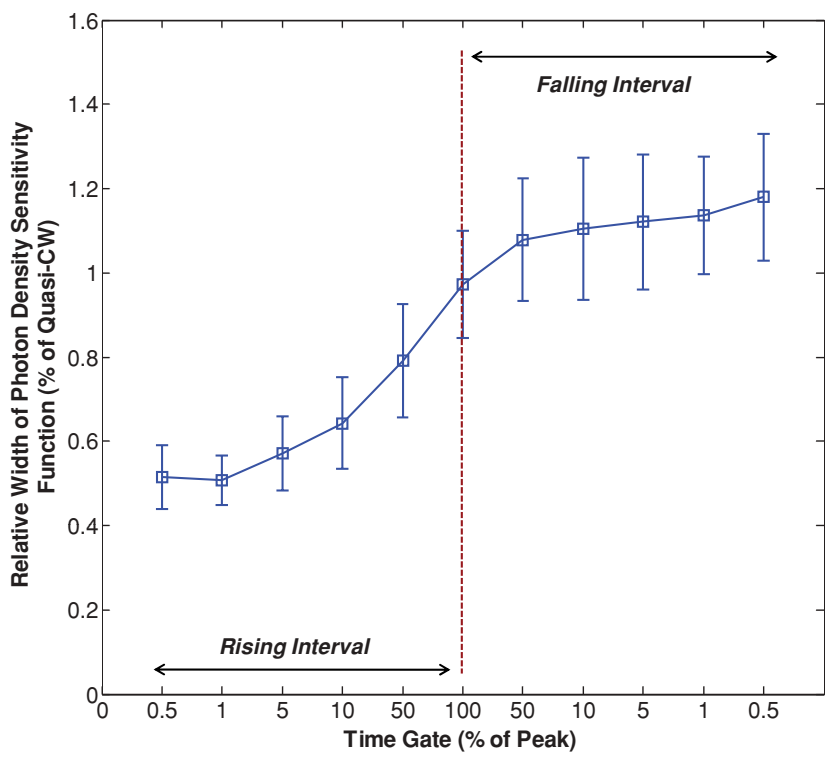

Fig. 5 Measured PDSF FWHM as a function of the quasi-CW width for a 2-mm-diam tube filled with Alexafluor-680. 


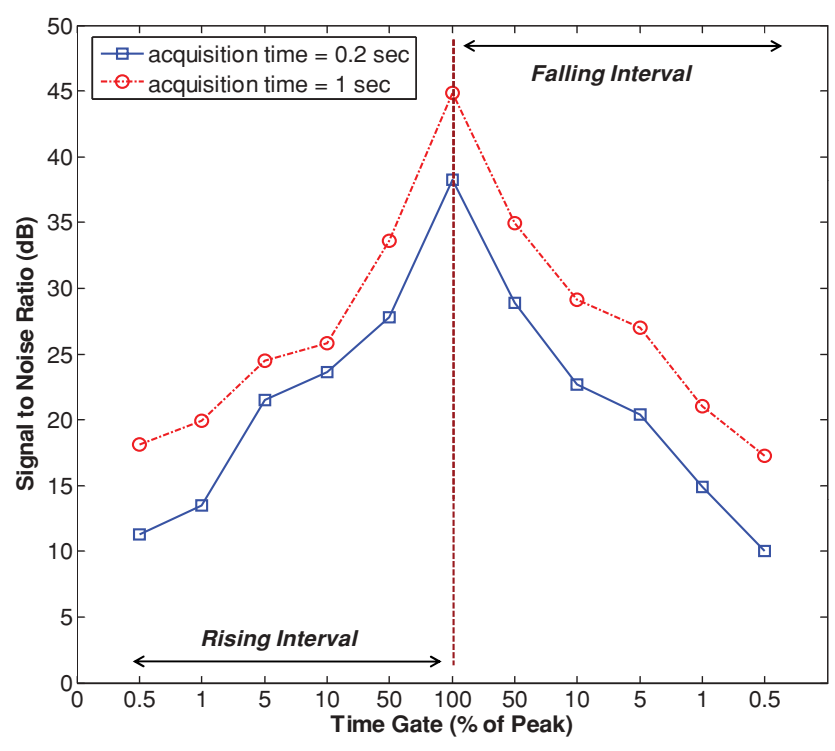

Fig. 6 SNR at different time gates for $0.2-$ and 1.0-s acquisition times. In both cases, the photon counting rate was $1 \times 10^{6}$ photons $/ \mathrm{s}$.

\subsection{Instrument Noise Performance at Different Time Gates}

Generally, it was observed that the uncertainty in the PDSF measurements was larger at early time gates due to increased noise at early time points. To quantify this, we performed 100 measurements with either 0.2- or 1-s total acquisition times, without an absorbing rod in place and determined the mean $(I)$ and standard deviation $(\sigma)$ of the intensity at each of the time points shown in Fig. 6. For these measurements, the photon count rate was $1 \times 10^{6}$ counts $/ \mathrm{s}$. Here, the chamber length was set to $2 \mathrm{~cm}, 1 \%$ Intralipid, and $50 \mathrm{ppm}$ ink $\left(\mu_{s}^{\prime}=17 \mathrm{~cm}^{-1}, \mu_{a}=0.15 \mathrm{~cm}^{-1}\right)$. The resulting SNR, defined as SNR $=20 \log (I / \sigma)$, is shown in Fig. 6. These data indicate that the SNR for photons arriving before the 5\% rise point of the time-resolved curve is less than $20 \mathrm{~dB}$, resulting in the larger measurement uncertainties. Therefore, while our other data show that measurement of "earlier" photons than about the 5 to $10 \%$ rise point marginally reduced the measured width of the PDSF, the system SNR performance was significantly worse. Note also that the measurement noise at very early time gates was about 2 times larger than would be expected from photon-counting (Poisson) noise. Analysis of our data shows that there appeared to be a small (5 to $10 \mathrm{ps)} \mathrm{low-}$ frequency wobble of the full-time curve during measurements. This had negligible effect at later time gates, but at very early times contributed to the reduced SNR.

\subsection{Theoretical Modeling}

Time-resolved Monte Carlo simulations were performed to model the experimental measurements already performed. Figure 7(a) shows the results of a simulation (parameters: 2-cm chamber length, $\left.\mu_{s}=112.5 \mathrm{~cm}^{-1}, g=0.85, \mu_{a}=0.15 \mathrm{~cm}^{-1}\right)$ compared to the experimentally measured data (Intralipid $=$ $1 \%$, ink $=50 \mathrm{ppm})$. The results show that the simulation results agreed well with the experimental data for the rising edge and quasi-CW data except at very early time gates $(<5 \%$ of the peak) where the simulation predicted a larger reduction in
PDSF FWHM than was experimentally observed. As we discuss shortly, this is most likely due to the non-ideal (i.e., finitewidth) temporal impulse response function of the system. Further, the simulations show that average number of photon scattering events for photons arriving in the $5 \%$ rising time point was about $1 / 3$ of the average number of all photons, i.e., $\sim 600$ and 2000 scatters, respectively. This is close to the decrease in instrument PDSF FWHM predicted from the simulation.

Finally, we used the time-resolved diffusion approximation (TRDA) to the BTE to calculate our instrument PDSF at early and quasi-CW time gates. The PDSF FWHM is shown in Fig. 7(b) along with the experimental data for the same conditions. From these data, we can see that the TRDA underestimates the reduction in the PDSF FWHM observed by measuring early photons by about $20 \%$ at the 5 and $10 \%$ rise points. The implications of the theoretical modeling are discussed shortly.

\section{Discussion and Conclusions}

In all cases investigated here, the use of early photons significantly reduced the amount of photon scatter and the corresponding instrument PDSF. Our data show that on average, about 40 to $60 \%$ reduction in the FWHM of the PDSF was achievable by measuring early compared to quasi-CW photons over the range of optical properties and path lengths investigated herein. This implies the volume of imaging PDSFs can be reduced by a factor of approximately 64 to $84 \%$ with a corresponding improvement in imaging resolution. The improvement is most likely highly dependent on the temporal response function of the measurement system; in this case, our system TIRF FWHM was 163 ps. The quantitative effect of altering instrument components and thus the system TIRF-for example, by replacing the filtered supercontinuum source with a faster pulsed laser-is not well understood and is the subject of ongoing study. Further, as we have demonstrated the reduction in photon scatter by measuring early photons comes at a cost of reduced noise performance and instrument sensitivity.

The optical properties and path lengths used here were chosen to be relevant for red and NIR light propagation in biological tissue. As noted, while increasing the path length, decreasing $\mu_{s}^{\prime}$ and $\mu_{a}$ all increased the absolute width of the PDSF for all time gates, the fractional reduction in FWHM was observed to be relatively consistent. This implies that measurement of early photons will give similar tomographic imaging improvement in different organs and tissue types in small animals. ${ }^{25,26}$ Further, the observed reduction in scatter at larger physical scales implies that the use early photons may indeed be valuable for tomographic imaging of larger laboratory animals or even at clinical scales.

Detection of "earlier" photons than about the 5 to $10 \%$ rise point of the time-resolved curve did not result in significantly narrower PDSFs, whereas the noise performance of the instrument was significantly worse. Therefore, we conclude that the 5 to $10 \%$ rise point is approximately the optimal operating point for early photon measurements for our system. This operating point is most likely highly instrument dependent, since source pulse width, detector temporal impulse response, detection sensitivity, and noise properties are different for each configuration.

With respect to modeling photon propagation, the diffusion approximation was found to agree well with our measured data 

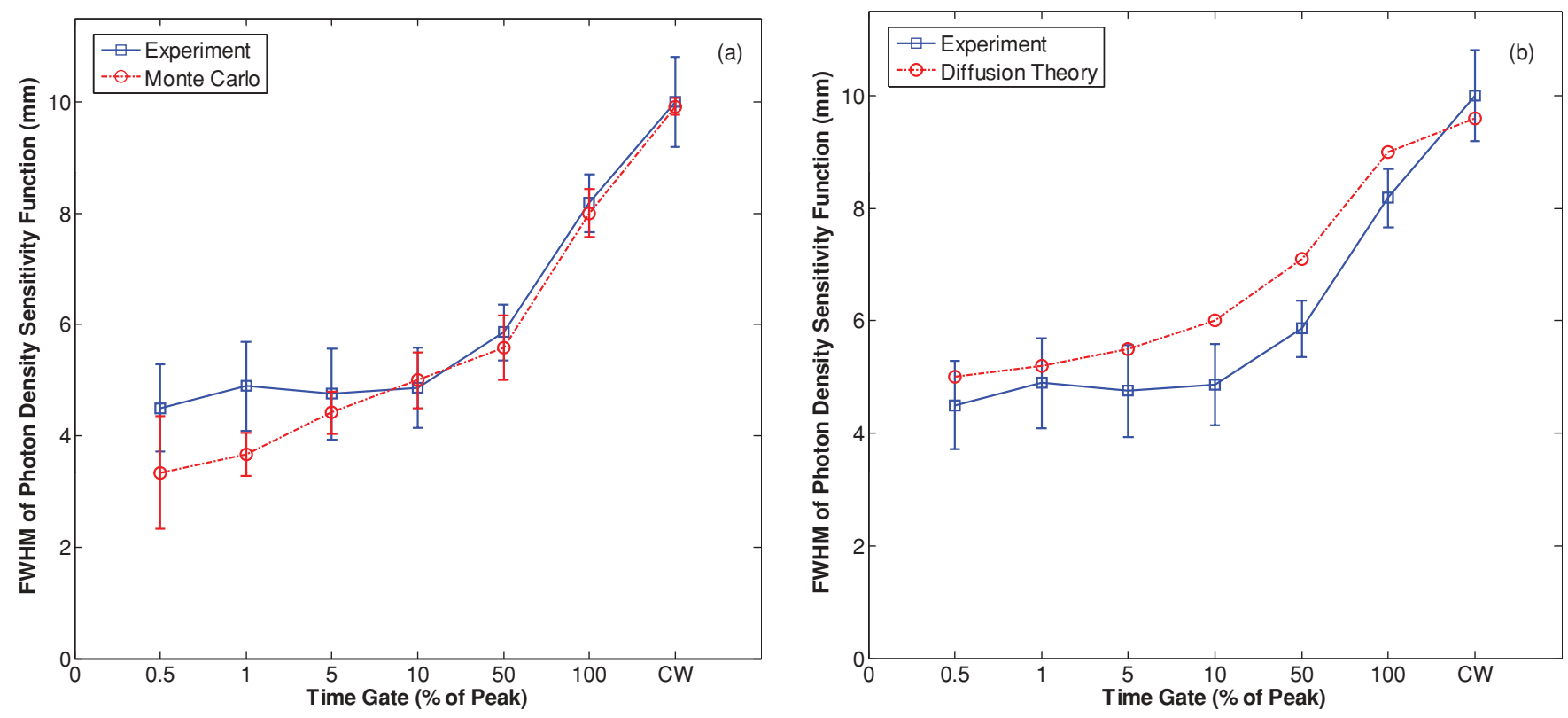

Fig. 7 Theoretical system PDSF FWHMs for the rising and quasi-CW portion of the curves calculated using (a) time-resolved Monte Carlo simulations and (b) the time-resolved diffusion approximation to the BTE.

for quasi-CW measurements but to underestimate the observed reduction in PDSF FWHM observed at early time gates (i.e., $<50 \%$ of the rise portion of the measured curve). While the discrepancy was small-about $20 \%$ overestimation at the 5 and $10 \%$ rise points - it was consistently observed versus our experimental data. This disagreement has been noted qualitatively previously. ${ }^{14,18}$ and is most likely due to the underlying P1 approximation as well as the assumption that sources emit isotropically. Therefore, we conclude that the TRDA will lead to data-model disagreements if used in the calculation of early photon forward models for the tomographic image reconstruction problem. Other models ${ }^{30}$ such as time-resolved P-3 or SP-N models or the full BTE were not evaluated here but may yield better data-model agreement over the complete time curve.

In contrast, time-resolved Monte Carlo simulations agreed well with our experimental data with the exception of very early time gates (earlier than the 5\% rise point). Simulations predict that a greater fractional reduction in the PDSF FWHM-by a factor of 4 or better-should be achievable, but we were not able to observe this with our setup. This is most likely due to the finite (non-ideal) TIRF of our system (FWHM of 163 ps) effectively "smearing" the reduction in scattering at very early times. However, alternative experimental configurations with a shorter TIRF may yield greater experimental improvement versus what was measured here. Again, the use of earlier time gates would most likely yield decreased measurement SNR, so that noise-resolution trade-offs would be an important consideration in the design of such an instrument.

\section{Acknowledgments}

This work was funded with a grant from the National Institutes of Health (R01EB012117-01) and from a Northeastern University laboratory startup grant. The assistance of Mr. Timothy Rossini in initial setup of the system is gratefully acknowledged.

\section{References}

1. A. H. Hielscher, "Optical tomographic imaging of small animals," Curr. Opin. Biotechnol. 16(1), 79-88 (2005).

2. V. Ntziachristos, C. H. Tung, C. Bremer, and R. Weissleder, "Fluorescence molecular tomography resolves protease activity in vivo," Nature Med. 8(7), 757-760 (2002).

3. A. Gibson and H. Dehghani, "Diffuse optical imaging," Philos. Transact. A Math. Phys. Eng. Sci. 367(1900), 3055-3072 (2009).

4. E. M. Hillman, "Optical brain imaging in vivo: techniques and applications from animal to man," J. Biomed. Opt. 12(5), 051402 (2007).

5. B. J. Tromberg, B. W. Pogue, K. D. Paulsen, A. G. Yodh, D. A. Boas, and A. E. Cerussi, "Assessing the future of diffuse optical imaging technologies for breast cancer management," Med. Phys. 35(6), 24432451 (2008).

6. U. J. Netz, J. Beuthan, and A. H. Hielscher, "Multipixel system for gigahertz frequency-domain optical imaging of finger joints," Rev. Sci. Instrum. 79(3), 034301 (2008).

7. D. Kepshire, N. Mincu, M. Hutchins, J. Gruber, H. Dehghani, J. Hypnarowski, F. Leblond, M. Khayat, and B. W. Pogue, "A microcomputed tomography guided fluorescence tomography system for small animal molecular imaging," Rev. Sci. Instrum. 80(4), 043701 (2009).

8. G. Zacharakis, J. Ripoll, R. Weissleder, and V. Ntziachristos, "Fluorescent protein tomography scanner for small animal imaging," IEEE Trans. Med. Imaging 24(7), 878-885 (2005).

9. N. C. Shaner, R. E. Campbell, P. A. Steinbach, B. N. Giepmans, A. E. Palmer, and R. Y. Tsien, "Improved monomeric red, orange and yellow fluorescent proteins derived from Discosoma sp. red fluorescent protein," Nature Biotechnol. 22(12), 1567-1572 (2004).

10. S. Ke, X. Wen, M. Gurfinkel, C. Charnsangavej, S. Wallace, E. M. Sevick-Muraca, and C. Li, "Near-infrared optical imaging of epidermal growth factor receptor in breast cancer xenografts," Cancer. Res. 63(22), 7870-7875 (2003).

11. J. T. Wessels, A. C. Busse, J. Mahrt, C. Dullin, E. Grabbe, and G. A. Mueller, "In vivo imaging in experimental preclinical tumor research-a review," Cytometry A 71(8), 542-549 (2007).

12. E. E. Graves, J. Ripoll, R. Weissleder, and V. Ntziachristos, "A submillimeter resolution fluorescence molecular imaging system for small animal imaging," Med. Phys. 30(5), 901-911 (2003).

13. A. H. Hielscher, A. Y. Bluestone, G. S. Abdoulaev, A. D. Klose, J. Lasker, M. Stewart, U. Netz, and J. Beuthan, "Near-infrared diffuse optical tomography," Dis. Markers 18(5-6), 313-337 (2002). 
14. M. Niedre and V. Ntziachristos, "Comparison of fluorescence tomographic imaging in mice with early arriving, and quasi-continuous-wave photons," Opt. Lett. 35(3), 369-371 (2010).

15. F. Leblond, H. Dehghani, D. Kepshire, and B. W. Pogue, "Earlyphoton fluorescence tomography: spatial resolution improvements and noise stability considerations," J. Opt. Soc. Am. A Opt. Image. Sci. Vis. 26(6), 1444-1457 (2009).

16. M. J. Niedre, R. H. de Kleine, E. Aikawa, D. G. Kirsch, R. Weissleder, and V. Ntziachristos, "Early photon tomography allows fluorescence detection of lung carcinomas and disease progression in mice in vivo," Proc. Natl. Acad. Sci. U.S.A. 105(49), 19126-19131 (2008).

17. G. M. Turner, G. Zacharakis, A. Soubret, J. Ripoll, and V. Ntziachristos, "Complete-angle projection diffuse optical tomography by use of early photons," Opt. Lett. 30(4), 409-411 (2005).

18. J. Wu, L. Perelman, R. R. Dasari, and M. S. Feld, "Fluorescence tomographic imaging in turbid media using early arriving photons and Laplace transforms," Proc. Natl. Acad. Sci. U.S.A. 94(16), 8783-8788 (1997).

19. A. T. Kumar, S. B. Raymond, A. K. Dunn, B. J. Bacskai, and D. A Boas, "A time domain fluorescence tomography system for small animal imaging," IEEE Trans. Med. Imaging 27(8), 1152-1163 (2008).

20. S. L. Jacques, "Time resolved propagation of ultrashort laser pulses within turbid tissues," Appl. Opt. 28(12), 2223-2229 (1989).

21. J. C. Hebden, A. Gibson, R. M. Yusof, N. Everdell, E. M. Hillman, D. T. Delpy, S. R. Arridge, T. Austin, J. H. Meek, and J. S. Wyatt, "Three-dimensional optical tomography of the premature infant brain," Phys. Med. Biol. 47(23), 4155-4166 (2002).
22. D. Grosenick, K. T. Moesta, H. Wabnitz, J. Mucke, C. Stroszczynski, R. Macdonald, P. M. Schlag, and H. Rinneberg, "Time-domain optical mammography: initial clinical results on detection, and characterization of breast tumors," Appl. Opt. 42(16), 3170-3186 (2003).

23. P. Taroni, L. Spinelli, A. Torricelli, A. Pifferi, G. M. Danesini, and R. Cubeddu, "Multi-wavelength time domain optical mammography," Technol. Cancer Res. Treat. 4(5), 527-538 (2005).

24. A. Bassi, C. D’Andrea, G. Valentini, R. Cubeddu, and S. Arridge, "Temporal propagation of spatial information in turbid media," Opt. Lett. 33(23), 2836-2838 (2008).

25. M. J. Niedre, G. M. Turner, and V. Ntziachristos, "Time-resolved imaging of optical coefficients through murine chest cavities," J. Biomed. Opt. 11(6), 064017 (2006).

26. G. Alexandrakis, F. R. Rannou, and A. F. Chatziioannou, "Tomographic bioluminescence imaging by use of a combined optical-PET (OPET) system: a computer simulation feasibility study," Phys. Med. Biol. 50(17), 4225-4241 (2005).

27. S. L. Jacques and B. W. Pogue, "Tutorial on diffuse light transport," J. Biomed. Opt. 13(4), 041302 (2008).

28. A. Kienle and M. S. Patterson, "Improved solutions of the steady-state and the time-resolved diffusion equations for reflectance from a semiinfinite turbid medium," J. Opt. Soc. Am. A Opt. Image. Sci. Vis. 14(1), 246-254 (1997).

29. L. Wang, S. L. Jacques, and L. Zheng, "MCML-Monte Carlo modeling of light transport in multi-layered tissues," Comput. Meth. Progr. Biomed. 47(2), 131-146 (1995).

30. A. D. Klose, "The forward and inverse problem in tissue optics based on the radiative transfer equation: a brief review," J. Quant. Spectrosc. Radiat. Transf. 111(11), 1852-1853 (2010). 